\title{
Model predictive control for intelligent speed adaptation in intelligent vehicle highway systems*
}

\author{
L.D. Baskar, B. De Schutter, and H. Hellendoorn
}

If you want to cite this report, please use the following reference instead:

L.D. Baskar, B. De Schutter, and H. Hellendoorn, "Model predictive control for intelligent speed adaptation in intelligent vehicle highway systems," Proceedings of the 17th IEEE International Conference on Control Applications, San Antonio, Texas, pp. 468-473, Sept. 2008.

Delft Center for Systems and Control

Delft University of Technology

Mekelweg 2, 2628 CD Delft

The Netherlands

phone: +31-15-278.24.73 (secretary)

URL: https://www.dcsc.tudelft.nl

*This report can also be downloaded via https://pub. deschutter.info/abs/08_011.html 


\title{
Model Predictive Control for Intelligent Speed Adaptation in Intelligent Vehicle Highway Systems
}

\author{
Lakshmi Dhevi Baskar, Bart De Schutter, and Hans Hellendoorn
}

\begin{abstract}
Intelligent Vehicle Highway Systems (IVHS) consist of automated highway systems in combination with intelligent vehicles and roadside controllers. The intelligent vehicles can communicate with each other and with the roadside infrastructure. The vehicles are organized in platoons with short intraplatoon distances, and larger distances between platoons. Moreover, all vehicles are assumed to be automated, i.e., throttle, braking, and steering commands are determined by an automated on-board controller. In this paper we first propose a model predictive control (MPC) approach to determine appropriate speeds for the platoons. Next, we discuss which prediction models are suited to be used as an on-line traffic prediction model in MPC for IVHS. The proposed approach is then applied to a simple simulation example in which the aim is to minimize the total time all vehicles spend in the network by optimally assigning speeds to the platoons.
\end{abstract}

\section{INTRODUCTION}

Due to the growing traffic demand and the growing need for mobility and transportation, traffic jams are still increasing in frequency, duration, and impact all around the world. Often traffic congestion problems can to some extent be solved by building new roads; however, this option is often not feasible due to lack of space, financial costs, or environmental constraints. An alternative, usually more feasible option is to use the existing infrastructure in a more efficient way through traffic management and traffic control. This approach combines advanced control methods with various control measures (such as traffic signals, ramp metering installations, dynamic speed limits, etc.) to reduce the impact of traffic jams.

As a next step in this direction, advanced technologies from the field of control theory, communication, and information technology are currently being combined with the existing transportation infrastructure and equipment. This will result in integrated traffic management and control systems, called Intelligent Vehicle Highway Systems (IVHS), that incorporate intelligence in both the roadside infrastructure and in the vehicles. Though this step is considered to be a long-term solution, this approach is capable of offering increased performance of the traffic system [1]-[3].

In order to diminish or even to eliminate the impact of driver reaction times and driver errors, complete control of driving tasks is preferred in IVHS-based traffic management systems. So in IVHS all vehicles are assumed to be fully

L.D. Baskar, B. De Schutter, and H. Hellendoorn are with the Delft Center for Systems and Control, Delft University of Technology, Delft, The Netherlands. email: $\{1$. d.baskar, j.hellendoorn $\}$ etudelft.nl, bedeschutter. info

B. De Schutter is also with the Marine \& Transport Technology department of Delft University of Technology. automated with throttle, braking, and steering commands being determined by an automated on-board controller. Such complete automation of the driving tasks allows to organize the traffic in platoons, i.e., a closely spaced group of vehicles traveling together with short intervehicle distances [4], [5]. In a platoon, the first vehicle is called the platoon leader and the other ones are said to be followers. Platoons travel at high speeds and to avoid collisions between platoons at these high speeds, a safe interplatoon distance of about $20-60 \mathrm{~m}$ should be maintained. Also, the vehicles in each platoon travel with small intraplatoon distances of about 2$5 \mathrm{~m}$, which are maintained by the automated on-board speed and distance controllers. By traveling at high speeds, by substantially eradicating human delays, and by maintaining short intraplatoon distances, the platoon approach allows more vehicles to travel on the network, which improves the traffic throughput [6], [7].

In this paper we will combine the intelligence of both roadside infrastructure and automated vehicles. More specifically, in the proposed approach, the roadside infrastructure will use traffic control and management methods that support platoons of intelligent vehicles. The control approach will also be embedded in the hierarchical traffic management and control framework for IVHS we have presented in [8]. In this paper, we will in particular concentrate on how the roadside controller can determine optimal speeds set-points for the platoons using model predictive control (MPC).

The paper is organized as follows. In Section II we present a short overview of intelligent vehicles (IVs) and IV-based control measures, with a particular focus on intelligent speed adaptation. Next, we discuss in Section III how MPC can be used to determine optimal speeds for the platoon leaders in IVHS. Section IV presents an overview of appropriate prediction models for use in IVHS-MPC. In Section V we apply the proposed approach to a case study based on simulations and we highlight the potential effects of IVHSMPC on the performance of the traffic system.

\section{INTELLIGENT VEHICLE HIGHWAY SYSTEMS (IVHS)}

\section{A. Intelligent vehicles and IV-based traffic control measures}

Intelligent Vehicles (IVs) are equipped with control systems that can sense the environment around the vehicle and that result in a more efficient vehicle operation by assisting the driver or by taking partial or complete control of the vehicle [9]. The platoon-based approach used in this paper assumes that all IVs are fully autonomous, i.e., complete control is taken of the vehicle operation. 


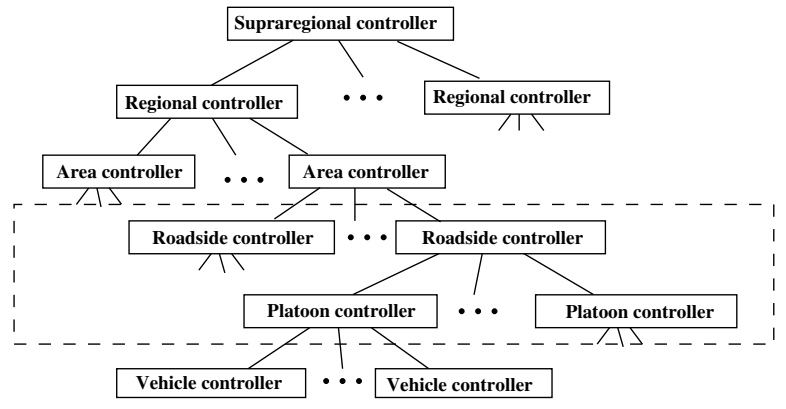

Fig. 1. IV-based framework of [8]. The focus of this paper is indicated by the dashed box.

There are several IV technologies that support and improve the platooning concept by allowing vehicle-vehicle and vehicle-roadside coordination [9], [10]:

- Intelligent Speed Adaptation (ISA),

- Adaptive Cruise Control (ACC),

- dynamic route planning and guidance.

In this paper we will focus on ISA and ACC.

ISA is based on a speed limiter incorporated within each vehicle that can take into account speed limit restrictions, that can adjust the maximum driving speed to the speed limit specified by the roadside infrastructure, and that can provide feedback to the driver or take autonomous action when that speed limit is exceeded. ISA systems could use fixed or dynamic speed limits. In the fixed case, the driver is informed about the speed limit, which could be obtained from a static database. Dynamic speed limits take into account the current road conditions such as bad weather, slippery roads, or major incidents before prescribing the speed limit.

An ACC system is a radar-based system that extends conventional cruise control and that is designed to monitor the immediate predecessor vehicle in the same lane, and to automatically adjust the speed of the equipped vehicle to match the speed of the preceding vehicle and to maintain a safe intervehicle distance [11]. Cooperative ACC is a further enhancement of ACC systems that uses wireless communication technologies to obtain real-time information about the speed, acceleration, etc. of the preceding vehicle. Vehicles equipped with cooperative ACC can exchange the information much quicker and allow to set the safe minimum time headway as small as $0.5 \mathrm{~s}$. Hence, with reduced headways between vehicles, the maximal traffic flow can be augmented even further.

\section{B. Hierarchical framework for IV-based traffic management}

In this section, we briefly present the hierarchical control framework for IVHS we have proposed in [8]. This framework distributes the intelligence between roadside infrastructure and vehicles, and uses IV-based control measures to prevent congestion and/or to improve the performance of the traffic network.

The control architecture of [8] is based on the platoon concept and consists of a multi-level control structure with local controllers at the lowest level and one or more higher supervisory control levels as shown in Figure 1. The layers of the framework can be characterized as follows:

- The higher-level controllers (such as area, regional, and supraregional controllers) provide network-wide coordination of the lower-level and middle-level controllers. The activities of a group of roadside controllers could be supervised by an area controller. In turn, a group of area controllers could be supervised or controlled by regional controllers, and so on.

- The roadside controllers use IV-based control measures to improve the traffic flow. A roadside controller may control a part of a highway, an entire highway, or a collection of highways. Each platoon in the highway network is considered as a one single entity to the roadside controller. This significantly reduces the complexity of the control problem compared to the case where each individual vehicle would be controlled by the roadside controller. As a consequence, the whole traffic network can be managed more efficiently.

The main tasks of the roadside controllers are to assign desired speeds for each platoon, safe distances to avoid collisions between platoons, desired platoon sizes depending on the traffic conditions, to provide dynamic route guidance for the platoons, ramp metering values at the on-ramps, and also to instruct for merges, splits, and lane changes of platoons.

- The platoon controllers receive commands from the roadside controllers and are responsible for control and coordination of each vehicle inside the platoon. The platoon controllers are mainly concerned with actually executing the interplatoon maneuvers (such as merges with other platoons, splits, and lane changes) and intraplatoon activities (such as maintaining safe intervehicle distances).

- The vehicle controllers present in each vehicle receive commands from the platoon controllers (e.g., set-points or reference trajectories for speeds (ISA), headways (ACC), and paths) and they translate these commands into control signals for the vehicle actuators such as throttle, braking, and steering actions.

In the remainder of the paper we will focus on the roadside controller and in particular on how optimal speed limits can be determined for the platoons.

\section{MODEL PREDICTIVE CONTROL FOR INTELLIGENT SPEED ADAPTATION IN IVHS}

\section{A. MPC for ISA}

Model Predictive Control (MPC) [12], [13] has originated in the process industry and it has already been successfully implemented for many industrial applications. MPC is based on (on-line) optimization and uses an explicit prediction model to determine the optimal values for the control measures (see Figure 2). The optimal control inputs are then applied to the system in a receding horizon approach. The receding horizon approach introduces a feedback mechanism, 


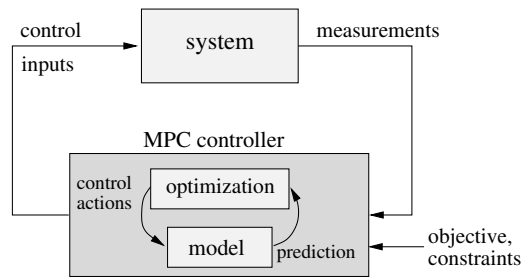

Fig. 2. Schematic representation of MPC.

which allows to reduce the effects of possible disturbances and mismatch errors.

We now explain how MPC can be applied for speed control in IVHS. MPC makes use of discrete-time models. Let $T_{\text {ctrl }}$ be the control sampling interval, i.e., the (constant) time interval between two updates of the control signal settings. At each time step $k$ (corresponding to the time instant $t=$ $k T_{\text {ctrl }}$ ), the roadside controller first measures or determines the current state $x(k)$ of the system. Recall that the roadside control works with platoons as basic entities. So in our case the state of the system includes the positions and speeds of the platoon leaders and the lengths of the platoons. Next, the controller uses an optimization algorithm in combination with a model of the system to determine the control inputs $u(k), \ldots, u\left(k+N_{\mathrm{p}}-1\right)$ that optimize a performance criterion $J(k)$ over a time interval $\left[k T_{\text {ctrl }},\left(k+N_{\mathrm{p}}\right) T_{\text {ctrl }}\right]$, where $N_{\mathrm{p}}$ is called the prediction horizon. In our case the control signal $u$ will consist of the speed limits for the platoon leaders.

There exists a wide range of traffic models [14]. An important factor that determines the choice of the model to be used in MPC is the trade-off between accuracy and computational complexity since at each time step $k$ the model will be simulated repeatedly within the on-line optimization algorithm. As a consequence, very detailed microscopic traffic simulation models are usually not suited as MPC prediction model. Instead, simplified or more aggregate models are usually applied. In Section IV we will present some models that are especially suited for use in MPC for IVHS. Note however that MPC is a modular approach so that in case a given prediction model does not perform well, it can easily be replaced by another prediction model.

Possible performance criteria $J(k)$ are the total time spent in a traffic network, the total throughput, the total fuel consumption, safety, or a combination of these. In this paper we will in particular consider the total time spent (TTS) by all the vehicles in the network:

$$
J_{\mathrm{TTS}}(k)=\sum_{j=0}^{N_{\mathrm{p}}} n_{\mathrm{veh}}(k+j) T_{\mathrm{ctrl}},
$$

where $n_{\mathrm{veh}}(k+j)$ is the number of vehicles that are present in the network at time $t=(k+j) T_{\text {ctrl }}$. Moreover, in order to prevent oscillations and frequent shifting in the control signals, one often adds a penalty on variations in the control signal $u$, which results in the total performance function

$$
J_{\text {tot }}(k)=J(k)+\alpha \sum_{j=0}^{N_{\mathrm{p}}}\|u(k+j)-u(k+j-1)\|_{2},
$$

where $\alpha>0$ is a weighting factor.

The MPC controller also explicitly takes into account operational constraints such as minimum separation between the platoons, minimum and maximum speeds, minimum headways, etc. To reduce the computational complexity of the problem, one often introduces a constraint of the form $u(k+j)=u(k+j-1)$ for $j=N_{\mathrm{c}}, \ldots, N_{\mathrm{p}}-1$, where $N_{\mathrm{c}}\left(<N_{\mathrm{p}}\right)$ is called the control horizon.

In MPC the control actions are applied in a receding horizon fashion. This is done by applying only the first control sample $u(k)$ of the optimal control sequence to the system. Next, the prediction horizon is shifted one step forward, and the prediction and optimization procedure over the shifted horizon are repeated using new system measurements.

\section{B. Optimization methods}

Solving the MPC optimization problem (i.e., computing the optimal control actions) is the most demanding operation in the MPC approach. In our case the MPC approach gives rise to nonlinear nonconvex optimization problems that have to be solved on-line. So a proper choice of optimization techniques that suit the nature of the problem has to be made. In our case global or multi-start local optimization methods are required such as multi-start sequential quadratic programming [15], pattern search [16], genetic algorithms [17], or simulated annealing [18].

\section{VEHICLE AND TRAFFIC MODELING}

Now we describe simplified traffic models for vehicles and for platoons that can be used as (part of the) prediction model within the MPC-based roadside controller.

\section{A. Traffic flow modeling}

In this paper, we deal with the longitudinal aspects of the driver tasks, which can be classified as follows:

- free-flow behavior,

- car-following behavior,

- stop-and-go behavior.

In free-flow behavior, the vehicles can travel at their desired speed (corresponding to the speed limit, e.g., $120 \mathrm{~km} / \mathrm{h}$ ). As the traffic demand increases, the vehicles start to follow their predecessors at closer distances and at reduced speeds (50$80 \mathrm{~km} / \mathrm{h}$ ). Once the capacity of the highway is being utilized at its maximum, then the vehicles move with stop-and-go movements $(0-40 \mathrm{~km} / \mathrm{h})$.

\section{B. Vehicle models}

We use general kinematics motion equations to describe the dynamics of the vehicles, which, after discretization leads to:

$$
\begin{aligned}
& x_{i}(\ell)=x_{i}(\ell-1)+v_{i}(\ell-1) T_{\text {sim }}+0.5 a_{i}(\ell-1) T_{\text {sim }}^{2} \\
& v_{i}(\ell)=v_{i}(\ell-1)+a_{i}(\ell-1) T_{\text {sim }}
\end{aligned}
$$

where $\ell$ is the simulation step counter, $T_{\text {sim }}$ the simulation time step, $x_{i}$ the longitudinal position of vehicle $i, v_{i}$ the speed of vehicle $i$, and $a_{i}$ the acceleration of vehicle $i$. The acceleration used in (3)-(4) is calculated according to the 
current driving situation as will be explained below. Also, the acceleration is limited between a maximum acceleration and a maximum (in absolute value) comfortable deceleration.

We first consider models for human drivers. Next, we discuss models for the intelligent vehicles and for the platoons. We conclude with a description of a phenomenon called capacity drop.

\section{Longitudinal models for human drivers}

The time headway $T_{\text {head }}$ of a vehicle is defined as the time difference between the passing of the rear ends of the vehicle's predecessor and the vehicle itself at a certain location. When there is no predecessor or when the time headway to the predecessor is larger than the critical time headway (e.g., $10 \mathrm{~s}$ ), then the vehicle is said to be in free-flow mode. Once the vehicle travels with a smaller time headway than the critical time headway to its predecessor, then the vehicle is said to be in car-following mode.

1) Free-flow model: The acceleration for free-flow driving conditions is determined by the delayed difference between the current speed and the reference speed:

$$
a_{i}(\ell)=K\left(v_{\mathrm{ref}, i}(\ell-\sigma)-v_{i}(\ell-\sigma)\right)
$$

where $K$ is the proportional constant, $v_{\mathrm{ref}, i}$ is the reference speed, and $\sigma$ is the reaction delay ${ }^{1}$. The reference speed can either be issued by roadside infrastructure or it can be driver's desired maximum speed.

2) Car-following model: As described in [19] there exist various types of car-following models such as stimulus response models [20], collision avoidance models [21], psychophysical models [22], and cellular automata models [23].

We will use a stimulus response model to describe the behavior of human drivers as this model is most often used and also easy to implement. Stimulus response models are based on the hypothesis that each vehicle accelerates or decelerates as a function of the relative speed and distance between the vehicle and its predecessor. In particular, the Gazis-HermanRothery (GHR) model [24] states that after a reaction delay, the follower vehicle $i$ accelerates or decelerates in proportion to the speed of the vehicle itself, to the relative speed with respect to its predecessor (vehicle $i+1$ ), and to the inverse of distance headway between them. The reference acceleration is thus given by

$$
a_{i}(\ell)=C v_{i}^{\beta}(\ell) \frac{\left(v_{i+1}(\ell-d)-v_{i}(\ell-d)\right)}{\left(x_{i+1}(\ell-d)-x_{i}(\ell-d)\right)^{\gamma}}
$$

where $C, \beta$, and $\gamma$ are the model parameters (possibly with different values depending on whether the vehicle is in a congested and uncongested driving situation), and $d$ is the driver delay ${ }^{2}$.

\footnotetext{
${ }^{1}$ We assume here that the reaction time $T_{\text {react }}$, which typically has a value of $1-1.2 \mathrm{~s}$, is an integer multiple of the simulation time step $T_{\text {sim }}$. So, $T_{\text {react }}=$ $\sigma T_{\text {sim }}$ with $\sigma$ an integer.

${ }^{2}$ Here we assume again that $T_{\text {delay }}$, which typically has a value of $1-1.2 \mathrm{~s}$, is an integer multiple of $T_{\text {sim }}$. So, $T_{\text {delay }}=d T_{\text {sim }}$ with $d$ an integer.
}

\section{Longitudinal models for intelligent vehicles}

In our approach, intelligent vehicles will use ACC and ISA measures and are arranged in platoons. We now discuss how the accelerations for the platoon leaders and for the follower vehicles within a platoon are calculated.

1) Platoon leader model: Platoon leaders have an enforced-ISA system and the calculation of the acceleration for the platoon leader is based on a simple proportional controller:

$$
a_{i}(\ell)=K_{1}\left(v_{\mathrm{ISA}}(\ell)-v_{i}(\ell)\right)
$$

where $K_{1}$ is the proportional constant, and $v_{\text {ISA }}$ is the reference ISA speed provided by the roadside controller.

2) Follower vehicle model: The follower vehicles will use their on-board ACC system to maintain short intraplatoon distances. The ACC algorithm consists of a combined speed and distance controller:

$$
a_{i}(\ell)=K_{2}\left(h_{\mathrm{ref}, i}(\ell)-\left(x_{i+1}(\ell)-x_{i}(\ell)\right)\right)+K_{3}\left(v_{i+1}(\ell)-v_{i}(\ell)\right)
$$

where $K_{2}$ and $K_{3}$ are constants, and $h_{\mathrm{ref}, i}$ is the reference distance headway for vehicle $i$. Note that the speed controller is based on the same principle as the one used in the platoon leader model, but with the platoon leader's speed as the reference speed. The distance controller calculates the safe distance headway as follows:

$$
h_{\mathrm{ref}, i}(\ell)=S_{0}+v_{i}(\ell) T_{\mathrm{head}, i}+L_{i}
$$

where $S_{0}$ is the minimum safe distance that is to be maintained at zero speed, $T_{\text {head }, i}$ is the time headway for vehicle $i$, and $L_{i}$ is the length of vehicle $i$.

\section{E. Platoon-based prediction model}

On a more aggregate level, we can also consider a platoon of vehicles as a single entity without taking the detailed interactions among the individual vehicles within a platoon into account. So essentially we consider a platoon as one "big vehicle" with a length that is a function of the speed of the platoon (due to the dependence of the intervehicle spacing managed by the ACC on the speed (cf. (9))), and of the number and lengths of the vehicles in the platoon. The dynamics equations for the speed and position of the platoon are the same as those of a platoon leader presented above. Consider platoon $p$ and assume for the sake of simplicity that the vehicles in the platoon are numbered 1 (last vehicle), 2 (one but last vehicle), $\ldots, n_{p}$ (platoon leader). The speed dependent length $L_{\text {plat, } p}(\ell)$ of platoon $p$ is then given by

$$
L_{\text {plat }, p}=\left(n_{p}-1\right)\left(S_{0}+S_{1} v_{n_{p}}(\ell)\right)+\sum_{i=1}^{n_{p}} L_{i},
$$

where $S_{0}+S_{1} v_{n_{p}}(\ell)$ is the speed-dependent intervehicle spacing between the vehicles in the platoon, with $S_{0}$ the minimum safe distance that is to be maintained at zero speed, $S_{1}$ a model constant, $v_{n_{p}}$ the speed of the platoon (leader), and $L_{i}$ the length of vehicle $i$. 


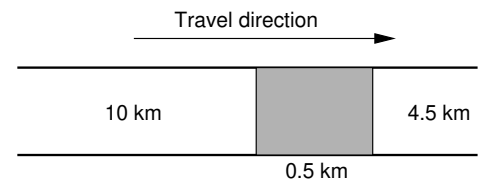

$15 \mathrm{~km}$ stretch

Fig. 3. Set-up of the case study.

\section{F. Capacity drop}

In general, traffic congestion occurs when the available network resources are not sufficient to handle the traffic demand (recurrent congestion), or due to irregular occurrences, such as traffic incidents (non-recurrent congestion). In practice, traffic jams or congestion result in capacity drop [25]. This phenomenon causes the expected maximum outflow from the jammed traffic to be less than in the case of free-flow traffic. This is mainly caused by the delay in reaction time and increased intervehicle distance (time headway) when vehicles start to exit from a traffic jam. For human drivers the capacity drop is typically of the order of $2-7 \%$. With fully automated vehicles the capacity drop can be reduced to almost $0 \%$.

\section{CASE STUdy}

In this section, we present a simple case study in which the MPC control strategy described in Section III is used by the roadside controller layer.

\section{A. Set-up}

As a test-bed for illustrating the proposed IVHS-MPC approach we use a basic set-up consisting of a $15 \mathrm{~km}$ singlelane highway stretch with one origin and one destination and without any intermediate on-ramps or off-ramps (see Figure 3). We will compare three different situations:

- uncontrolled traffic (with human drivers),

- controlled traffic with human drivers and autonomous ISA as control measure,

- IV-based traffic control with platoons.

For the sake of simplicity all vehicles are assumed to be of the same length $\left(L_{i}=4 \mathrm{~m}\right)$. For the situation with human drivers and ISA we assume that ISA limits the speed in a hard way and that human drivers cannot surpass the imposed speed limit. In the IV-based case with platoons we assume that all the vehicles are fully automated IVs equipped with advanced communication and detection technologies such as in-vehicle computers and sensors, and with on-board ACC and ISA controllers.

\section{B. Scenario}

We simulate a period of $30 \mathrm{~min}$ starting at time $t_{\text {start }}=$ $7 \mathrm{~h} 20 \mathrm{~min}$ and ending at time $t_{\text {end }}=7 \mathrm{~h} 50 \mathrm{~min}$. The total demand of vehicles is $1600 \mathrm{veh} / \mathrm{h}$. In the proposed scenario an incident occurs at position $x=10 \mathrm{~km}$ over a length of $0.5 \mathrm{~km}$, and it exists for a time interval of $15 \mathrm{~min}$, starting at time $t_{1}=t_{\text {start }}=7 \mathrm{~h} 20 \mathrm{~min}$ and ending at time $t_{2}=7 \mathrm{~h}$ 35 min. During this interval, the maximum outflow from the incident position $x$ is less when compared to free-flow traffic due to the capacity drop. The value of this capacity drop in our case is around $7 \%$ for human drivers (both in the controlled and the uncontrolled case) and around $0 \%$ for platoons (due to the full automation). After time $t_{2}$, the traffic flow at position $x$ returns slowly to its regular value.

\section{Models}

As indicated above we are interested in comparing the simulation results obtained for the same scenario using human driving (both without and with control) and using our platoon-based hierarchical approach. For this purpose, we have developed simulation models in Matlab for human driving and platoon driving. For the sake of simplicity and to avoid calibration, we have used the same models for both simulation and prediction purposes in this simulation study.

For the vehicle models we have used (3)-(4) with the reference accelerations given by respectively (5)-(6) for uncontrolled human drivers, (5)-(6) with $v_{\text {ref }, i}(\ell)$ equal to the ISA speed limit for human drivers with ISA, and (7)-(9) for platoons of intelligent vehicles. If we express distances in $\mathrm{m}$, times in $\mathrm{s}$, and speeds in $\mathrm{m} / \mathrm{s}$, the values of the various parameters in these models have the following values. For the car-following model (6) we have ${ }^{3} C=1.55, \beta=1.08$, and $\gamma=1.65$ for uncongested driving, and $C=2.55, \beta=-1.67$, and $\gamma=-0.89$ for congested driving. Furthermore, we have selected $\sigma=1, d=1$, and $K=K_{1}=\frac{1}{60}$. For the follower vehicle model (8)-(9) we have $K_{2}=1.58, K_{3}=1.8$, and $S_{0}=2$ for all vehicles.

The time step $T_{\text {sim }}$ for the simulations is set to $1 \mathrm{~s}$.

\section{Control problem}

The goal of our traffic controller is to improve the traffic performance. The objective that we consider is minimization of the total time spent (TTS) by all the vehicles in the network (see (1)) using dynamic speed limits as the control handle. So the control signal $u$ for the MPC problem of time step $k$ includes speed limits for all platoons that are in the network at time step $k$. We have also included a penalty term (cf. (2)) with $\alpha=0.02$. We consider a maximum speed of $120 \mathrm{~km} / \mathrm{h}$ for both the human drivers and the platoon leaders. As we focus on dynamic speed limits for each platoon, the platoon size is not yet considered to be a control variable, but it is kept fixed at 5 for all platoons.

The control sampling time $T_{\mathrm{c}}$ is set at $1 \mathrm{~min}$. For the prediction horizon $N_{\mathrm{p}}$ we have taken a value that corresponds to $15 \mathrm{~min}$, and for the control horizon $N_{\mathrm{c}}$ we have selected a value that corresponds to $3 \mathrm{~min}$ so as to limit the number of optimization variables.

For solving the MPC optimization problems we have used the patternsearch command incorporated in the Genetic Algorithm and Direct Search Toolbox of Matlab.

\section{E. Results and analysis}

For the scenario discussed above, a closed-loop MPC simulation has been carried out. The total time spent by

\footnotetext{
${ }^{3}$ These values are the same as the ones used in MITSIM [26].
} 


\begin{tabular}{|l|c|c|}
\hline Case & TTS (veh.h) & Relative improvement \\
\hline uncontrolled case & 141.11 & $0 \%$ \\
controlled (human drivers) & 118.86 & $15.77 \%$ \\
controlled (platoons) & 93.83 & $33.25 \%$ \\
\hline
\end{tabular}

TABLE I

RESUlTS OF THE THREE APPROACHES. THE TTS IS THE TOTAL TIME SPENT BY ALL VEHICLES IN THE NETWORK DURING THE ENTIRE SIMULATION PERIOD.

all the vehicles in the network during the period $\left[t_{\mathrm{start}}, t_{\mathrm{end}}\right]$ (nearly 465 vehicles are generated during this period) is calculated for human driving with ISA control and without control, and for IV-based platoons with speed limit control. The results are reported in Table I.

The relative improvement is computed with respect to the uncontrolled case. Clearly, the IV-based traffic with platoons results in the best performance with an improvement of about $33 \%$ with respect to the uncontrolled case. The results can be explained as follows.

In the uncontrolled case with human drivers, when there are no vehicles in front of the driver or if there is enough space between two drivers, the drivers maintain their desired speed. But when a driver is confronted with an incident, he has to decelerate in order to avoid a collision and he has to wait until the incident gets cleared. This results in a large time spent in the network for that vehicle, and thus also in a higher value of the TTS for the entire simulation period.

For the same scenario but with the human driver and ISA control, the MPC approach can predict the presence of the incident and prevent it or diminish its negative impacts by slowing down the vehicles before they reach the incident. For the platoon-based approach there is an additional performance improvement caused by the full automation, which allows to maintain small intervehicle distances (so that more cars are allowed to traverse the network more quickly) and which results in an almost $0 \%$ capacity drop.

\section{CONCLUSIONS AND FUTURE RESEARCH}

We have presented how model predictive control (MPC) can be used to determine optimal speeds for platoons in an intelligent vehicle highway system. The proposed approach has been illustrated using a case study based on simulations. The results of the case study highlight the potential benefits and improvements that can be obtained by using MPC for intelligent speed adaptation in IVHS. Future research topics include: additional and more extensive case studies, inclusion of additional control measures apart from speed limits, explicit consideration of the other levels in the IVHS control hierarchy of [8], and extension to larger networks.

\section{ACKNOWLEDGMENTS}

Research supported by the VIDI project "Multi-Agent Control of Large-Scale Hybrid Systems" (DWV.6188) of the Dutch Technology Foundation STW, Applied Science division of NWO and the Technology Programme of the Dutch Ministry of Economic Affairs, the BSIK projects
"Transition to Sustainable Mobility (TRANSUMO)" and "Next Generation Infrastructures (NGI)", the Transport Research Centre Delft, and the Delft Research Center Next Generation Infrastructures.

\section{REFERENCES}

[1] J. M. Sussman, "Intelligent vehicle highway systems: Challenge for the future," IEEE Micro, vol. 1, no. 14-18, pp. 101-104, Jun. 1993.

[2] R. K. Jurgen, "Smart cars and highways go global," IEEE Spectrum, vol. 28, no. 5, pp. 26-36, May 1991.

[3] R. E. Fenton, "IVHS/AHS: Driving into the future," IEEE Control Systems Magazine, vol. 14, no. 6, pp. 13-20, Dec. 1994.

[4] P. Varaiya, "Smart cars on smart roads," IEEE Transactions on Automatic Control, vol. 38, no. 2, pp. 195-207, Feb. 1993.

[5] S. Shladover, C. A. Desoer, J. K. Hedrick, M. Tomizuka, J. Walrand, W. B. Zhang, D. H. McMahon, H. Peng, S. Sheikholeslam, and N. McKeown, "Automatic vehicle control developments in the PATH program," IEEE Transactions on Vehicle Technology, vol. 40, no. 1, pp. 114-130, Feb. 1991.

[6] M. Broucke and P. Varaiya, "The automated highway system: A transportation technology for the 21st century," Control Engineering Practice, vol. 5, no. 11, pp. 1583-1590, Nov. 1997.

[7] K. Li and P. Ioannou, "Modeling of traffic flow of automated vehicles," IEEE Transactions on Intelligent Transportation Systems, vol. 5, no. 2, pp. 99-113, Jun. 2004.

[8] L. Baskar, B. De Schutter, and H. Hellendoorn, "Hierarchical traffic control and management with intelligent vehicles," in Proceedings of the 2007 IEEE Intelligent Vehicles Symposium (IV'07), Istanbul, Turkey, Jun. 2007, pp. 834-839.

[9] R. Bishop, Intelligent Vehicles Technology and Trends. Artech House, 2005.

[10] L. S. Comte, "New systems: new behaviour?" Transportation Research Part F, vol. 3, no. 2, pp. 95-111, May 2000.

[11] L. C. Davis, "Effect of adaptive cruise control systems on traffic flow," Physical Review E, vol. 69, pp. 1-8, 2004.

[12] J. M. Maciejowski, Predictive Control with Constraints. Harlow, England: Prentice Hall, 2002.

[13] E. F. Camacho and C. Bordons, Model Predictive Control in the Process Industry. Berlin, Germany: Springer-Verlag, 1995.

[14] C. Daganzo, Fundamentals of Transportation and Traffic Operations. Pergamon Press, 1997.

[15] P. M. Pardalos and M. G. C. Resende, Handbook of Applied Optimization. Oxford, UK: Oxford University Press, 2002.

[16] C. Audet and J. E. Dennis Jr., "Analysis of generalized pattern searches," SIAM Journal on Optimization, vol. 13, no. 3, pp. 889903, 2007.

[17] L. Davis, Handbook of Genetic Algorithms. New York: Van Nostrand Reinhold, 1991.

[18] R. W. Eglese, "Simulated annealing: A tool for operations research," European Journal of Operational Research, vol. 46, pp. 271-281, 1990.

[19] M. Brackstone and M. McDonald, "Car-following: A historical review," Transportation Research Part F, vol. 2, no. 4, pp. 181-196, 1999.

[20] A. May, Traffic Flow Fundamentals. Englewood Cliffs, New Jersey: Prentice-Hall, 1990.

[21] E. Kometani and T. Sasaki, "Dynamic behaviour of traffic with a nonlinear spacing speed relationship," in Proceedings of the Symposium for Theory Traffic Flow, Research Laboratories, General Motors, New York, 1959, pp. 105-109.

[22] R. M. Michaels, "Perceptual factors in car following," in Proceedings of the 2nd International Symposium for Theory Road Traffic Flow, Paris, France, 1963, pp. 44-59.

[23] K. Nagel, "Particle hopping models and traffic flow theory," Physical Review E, vol. 53, pp. 4655-4672, 1996.

[24] D. Gazis, R. Herman, and R. Rothery, "Nonlinear follow the leader models of traffic flow," Operations Research, vol. 9, no. 4, pp. 545567, Jun. 1961

[25] F. L. Hall and K. Agyemang-Duah, "Freeway capacity drop and the definition of capacity," Transportation Research Record, no. 1320, pp. 91-98, 1991.

[26] Q. Yang and H. N. Koutsopoulos, "A microscopic traffic simulator for evaluation of dynamic traffic management systems," Transportation Research Part C, vol. 4, no. 3, pp. 113-129, 1996. 